\title{
La inteligencia emocional y su calidad de vida durante la pandemia Covid-19 en personas de la tercera edad del hogarde acogimiento del adulto mayor del cantón Cevallos
}

Emotional intelligence and quality of life during the Covid-19 pandemic in elderly people from the foster home for the elderly in Cevallos

1 Valeria Lissette Campaña Chaglla $\quad$ (iD) https://orcid.org/0000-0003-0718-1011 Universidad Técnica de Ambato, Facultad de Ciencias Humanas y de la educación, Carrera de Psicopedagogía. Ambato; Ecuador. vcamapana5082@uta.edu.ec

2 Tannia Alejandra Martínez Ortiz https://orcid.org/0000-0001-7873-6745 Universidad Técnica de Ambato, Facultad de Ciencias Humanas y de la educación, Carrera de Psicopedagogía. Ambato; Ecuador tmartinez6678@uta.edu.ec

3 Roberto Enrique Alvarado Quinto iD https://orcid.org/0000-0002-0047-7779 Universidad Técnica de Ambato, Facultad de Ciencias de la Educación, Carrera de psicopedagogía. Ambato, Ecuador. re.alvarado@uta.edu.ec

4 Diana Alexandra Molina Peña https://orcid.org/0000-0002-8902-4433

Universidad Técnica de Ambato, Facultad de Ciencias Humanas y de la educación, Carrera de Psicopedagogía. Ambato; Ecuador

Artículo de Investigación Científica y Tecnológica Enviado: $24 / 12 / 2021$

Revisado: $29 / 12 / 2021$

Aceptado: $12 / 01 / 2022$

Publicado:08/03/2023

DOI: https://doi.org/10.33262/concienciadigital.v6i1.4.2014

Cítese: Campaña Chaglla, V. L., Martínez Ortiz, T. A., Alvarado Quinto, R. E., \& Molina Peña, D. A. (2023). La inteligencia emocional y su calidad de vida durante la pandemia Covid19 en personas de la tercera edad del hogar de acogimiento del adulto mayor del cantón Cevallos. ConcienciaDigital, 6(1.4), 566-580. https://doi.org/10.33262/concienciadigital.v6i1.4.2014

CONCIENCIA DIGITAL, es una Revista Multidisciplinar, Trimestral, que se publicará en soporte electrónico tiene como misión contribuir a la formación de profesionales competentes con visión humanística y crítica que sean capaces de exponer sus resultados investigativos y científicos en la misma medida que se promueva mediante su intervención cambios positivos en la sociedad. https://concienciadigital.org

La revista es editada por la Editorial Ciencia Digital (Editorial de prestigio registrada en la Cámara Ecuatoriana de Libro con No de Afiliación 663) www.celibro.org.ec 


\section{Palabras claves:} inteligencia emocional, calidad de vida, habilidades, relación interpersonal, comunicación, sociedad, desarrollo personal.

Keywords: emotional intelligence, quality of life, skills, interpersonal relationship, communication, society, personal development.

\section{Resumen}

El presente artículo se inscribe en el contexto de la investigación en curso "La inteligencia emocional y su calidad de vida durante la pandemia Covid -19 en personas de la tercera edad del hogar de acogimiento del adulto mayor del cantón Cevallos" de la dirección de la Investigación y Desarrollo (DIDE) Universidad Técnica de Ambato, Ecuador. La investigación aborda sobre la inteligencia emocional y la calidad de vida, muestra que la necesidad de desarrollar resiliencia en los adultos mayores es un problema para que puedan lidiar con otros problemas de la vida cotidiana; Así también tienen inteligencia emocional para controlar sus propias emociones y reconocer los sentimientos de los demás, lo que les permite mantener relaciones sociales saludables. El objetivo del estudio fue analizar la relación entre inteligencia emocional y calidad de vida de ancianos en un asilo de ancianos en San Pedro de Cevallos, y el método de investigación aplicado fue cuantitativo - cualitativo, con el método bibliográfico y nivel relativo de caracterización; como método, se prepara un test para los alumnos y una encuesta para los profesores. Los resultados identificaron una mayor resiliencia e inteligencia emocional para los adultos mayores, con técnicas y estrategias específicas para lograr una mayor felicidad personal, enfatizando el conocimiento sobre sus habilidades y destrezas, el uso apropiado y control del tiempo y las emociones desarrollar dimensiones de atención y claridad emocionales.

\section{Abstract}

This article is part of the context of the ongoing investigation "Emotional intelligence and quality of life during the covid -19 pandemic in elderly people in the foster home for the elderly in Cevallos" of the direction of the Research and Development (DIDE) Technical University of Ambato, Ecuador. The research work refers to emotional intelligence and its quality of life, where the need to develop resilience in older adults has been shown as problematic so that they can face various problems of daily life; and thus, also the emotional intelligence to maintain control of their emotions and recognize those of others, allowing them to maintain healthy social relationships. The research objective set out to analyze the relationship between emotional intelligence and quality of life of "Hogar de Acogomiento del adulto mayor de San Pedro 


\begin{abstract}
de Cevallos", the research approach taken was quantitativequalitative, with the bibliographic modality and correlational descriptive level; As a technique, a test was established for the students and a survey for the teachers. The results achieved to determine the strengthening of the resilience and emotional intelligence of older adults, with specific techniques and strategies to obtain greater personal well-being, focusing on the knowledge of their abilities and skills, the use and control of time appropriately, and as regards to the emotions develop the dimensions of emotional attention and emotional clarity.
\end{abstract}

\title{
Introducción
}

En las personas adultas mayores, la investigación emocional y cómo afectan la adaptación y la calidad de vida es fundamental. Por esta razón, la importancia de la inteligencia emocional y la calidad de vida es público, sabiendo que las personas mayores son importantes para nuestra sociedad, y es necesario garantizar el estado emocional y tener la atención necearía.

Necesitamos saber que la inteligencia emocional desde diferentes puntos son la capacidad de motivarse, será un muy buen ejemplo para lograr una estabilidad emocional completa en la persona adulta mayor. Cuando hablamos de la estabilidad emocional, nos referimos a las personas que pueden comprender la naturaleza de sus propias emociones, conocer su origen, expresarlos o controlarlos de acuerdo con los requisitos e incluso para predecir sus propios sentimientos.

Según Velongia (1994), menciona que la calidad de vida de los ancianos es el resultado de la interacción entre las diferentes características de la existencia humana, cada característica contribuye de una u otra manera para permitir una felicidad óptima, aplicando el proceso de evolución del envejecimiento, la adaptación de los individuos para cambios biológicos y psicólogos, se proporcionan individualmente y de manera diferente; Adaptación a la salud física, fallas de memoria y miedo, abandono, mortalidad, dependencia o discapacidad.

La investigación actual ha sido instruida para determinar la inteligencia emocional que puede ser un factor que influye la calidad de vida, presentando así un concepto de una guía metódica en la resiliencia para lograr llegar a un bienestar correcto y un envejeciendo activo.

\section{Metodología}

Materiales: Los materiales y recursos utilizados se describen a continuación: 


\section{Tabla 1}

Recursos materiales, institucionales, humanos, financieros

\begin{tabular}{|c|c|c|}
\hline $\begin{array}{l}\text { DETALLE } \\
\text { Recursos Institucionales }\end{array}$ & CANTIDAD & VALOR \\
\hline $\begin{array}{l}\text { Biblioteca Virtual de la Universidad } \\
\text { "Hogar de Acogimiento del adultomayor del Cantón } \\
\text { Cevallos" }\end{array}$ & - & $\begin{array}{l}- \\
-\end{array}$ \\
\hline \multicolumn{3}{|l|}{ Recursos Humanos } \\
\hline Tutor de tesis & - & - \\
\hline Coordinación de la carrera de Psicopedagogía & - & - \\
\hline $\begin{array}{l}\text { Personas de la tercera edad del "Hogar de Acogimiento } \\
\text { del adulto mayor del Cantón Cevallos" }\end{array}$ & - & - \\
\hline \multicolumn{3}{|l|}{ Recursos Materiales } \\
\hline Resmas de Hojas & 2 & $\$ 8$ \\
\hline Impresiones y copias & - & $\$ 30$ \\
\hline Test TMMS-24 de inteligenciaemocional & 1 & $\$ 15$ \\
\hline Cuestionario WHOQOL-BREF de calidad de vida & 1 & $\$ 15$ \\
\hline Microsoft Word & Libre & $\$ 0$ \\
\hline Microsoft Excel & Libre & $\$ 0$ \\
\hline Google Forms & Libre & $\$ 0$ \\
\hline \multicolumn{3}{|c|}{ Recursos Financieros } \\
\hline Transporte & & $\$ 40$ \\
\hline Alimentación & & $\$ 40$ \\
\hline \multirow[t]{3}{*}{ Imprevistos } & & $\$ 30$ \\
\hline & Presupuesto: & $\$ 178$ \\
\hline & Total: & $\$ 178$ \\
\hline
\end{tabular}

Fuente: Molina (2021)

El proyecto ofrece un enfoque cualitativo y cuantitativo, porque no se centra en un enfoque integral, sino que mediante la recopilación de información se obtendrán resultados sobre los problemas que enfrentan las personas mayores en la actualidad, y se obtendrán datos estadísticos e información sobre la realidad emocional y cualitativa. de la vida que están atravesando, lo que nos ayudará a definir el problema de manera más específica.

La investigación se encuentra en un nivel exploratorio, donde se utilizarán metodologías que aporten datos y puedan abordar aspectos necesarios para determinar la información necesaria para encontrar una solución a la hipótesis en cuestión.

Es descriptivo porque identificará los aspectos individuales y psicológicos de las personas adultas mayores para estructurar los parámetros a encontrar, y así detallar y cuantificar los datos encontrados mediante técnicas de investigación aplicada.

Este estudio es del tipo correlacional, porque se determinará el grado de relación entre la inteligencia emocional y la calidad de vida del adulto mayor.

El presente estudio da una base teórica porque se trabaja con procesos de análisis de datos, con la aplicación de pruebas y test, se interpretará la información a través de la 
documentación existente, con el propósito de identificar aspectos significativos en los adultos mayores, los test son de carácter científicos - bibliográficos con el fin de proporcionar información veraz.

Por la situación actual del covid-19 la información recopilara de la realizara de manera virtual, con un estudio de campo a través de la aplicación del Test TMMS-24 de inteligencia emocional y el Cuestionario WHOQOL-BREF de calidad de vida en los adultos mayores del "Hogar de Acogimiento Del Adulto Mayor de San Pedro de Cevallos".

Para Cortes y otro, se entiende por "población a la totalidad del universo, a personas que cumplen las mismas características con significancia para estudiar, tomando en cuenta una clara definición para que en todo momento se sepa que elementos lo componen" (p. 20)

La población con la cual se realizó la presente investigación está compuesta por 20 las personad de la tercera del "Hogar De Acogimiento Del Adulto Mayor de San Pedro de Cevallos"

\section{Tabla 2}

Población "Hogar de acogimiento del adulto mayor de San Pedro de Cevallos"

\begin{tabular}{ccc}
\hline GENERO & CANTIDAD & PORCENTAJE \\
\hline Hombres & 16 & $80 \%$ \\
Mujeres & 4 & $20 \%$ \\
\hline
\end{tabular}

Fuente: Molina (2021)

La prueba del Trait Meta Mood Scale (TMMS-24), tuvo como propósito evaluar la inteligencia emocional intrapersonal percibida (atención, claridad y reparación emocional).

Se trató de un instrumento compuesto por 24 ítems que están diseñados con una escala de tipo Likert de cuatro puntos ( $1=$ nada de acuerdo, $2=$ algo de acuerdo $3=$ bastante de acuerdo y $4=$ totalmente de acuerdo). La calificación se obtuvo con la suma de los puntajes por cada dimensión (atención, claridad y reparación); y al sumarse los puntajes totales de las tres dimensiones se obtuvo el índice de la inteligencia emocional (Estrada et al., 2010).

Tabla 3

Dimensiones del Cuestionario WHOQL-BREF

\begin{tabular}{ll}
\hline Dimensión & Ítems \\
\hline Física & 7 \\
Psicológica & 6 \\
\hline
\end{tabular}


Tabla 3

Dimensiones del Cuestionario WHOQL-BREF (continuación)

\begin{tabular}{ll}
\hline $\begin{array}{l}\text { Relaciones } \\
\text { Sociales }\end{array}$ & 3 \\
Ambientales & 8 \\
Escala del test & 24 \\
\hline
\end{tabular}

Fuente: Molina (2021)

\section{Hipótesis}

La inteligencia emocional se relaciona con la calidad de vida en las personas de la tercera edad del "Hogar de Acogimiento el Adulto Mayor de San Pedro de Cevallos".

Para el cálculo de la hipótesis se aplicó el Test TMMS-24 para conocer cómo se encuentra su inteligencia emocional y cómo influye la misma en la calidad de vida de un Adulto Mayor.

Así mismo se aplicó el Cuestionario WHOQL-BREF para conocer como esta su calidad de vida y así poder desarrollar con los resultados presentados una Guía Metodológica para La Resiliencia en Adultos Mayores.

\section{Resultados}

Análisis y discusión de los resultados del Test de "Inteligencia Emocional"

\section{Tabla 4}

Escala de Fiabilidad del Instrumento "Inteligencia Emocional"

\begin{tabular}{ccc}
\hline Alfa de Cronbach & Población & Ítems \\
\hline 0,93 & 20 & 24 \\
\hline
\end{tabular}

Fuente: Personas de la tercera edad del "Hogar de Acogimiento Del Adulto Mayor de San Pedro De Cevallos. Formularios en línea; IBM SPSS

Elaborado: Molina (2021)

El Test de Inteligencia Emocional contiene 24 elementos los mismos fueron aplicados a 20 personas de la tercera edad del "Hogar de Acogimiento Del Adulto Mayor de San Pedro de Cevallos" evidenciándose un Alfa de Cronbach de 0,93, el cual conforme la tabla de escala de fiabilidad es excelente.

A continuación, se realiza un análisis de las 3 dimensiones que evalúa el test: 
Dimensión de Atención Emocional

Tabla 5

Escala de Fiabilidad de la Dimensión de Atención Emocional

\begin{tabular}{ccc}
\hline Alfa de Cronbach & Media & Ítems \\
\hline 0,93 & 49,88 & 8
\end{tabular}

Fuente: Personas de la tercera edad del "Hogar de Acogimiento Del Adulto Mayor de San Pedro De Cevallos. Formularios en línea; IBM SPSS

Elaborado: Molina (2021)

La Dimensión de Atención Emocional, es constituida por 8 elementos, evidenciándose un Alfa de Cronbach de 0,93 el cual conforme la tabla de escala de fiabilidad es "excelente", en cuanto a la media es de 49,88.

\section{Figura 1}

\section{Diagramas de barras - Dimensión de Atención Emocional}

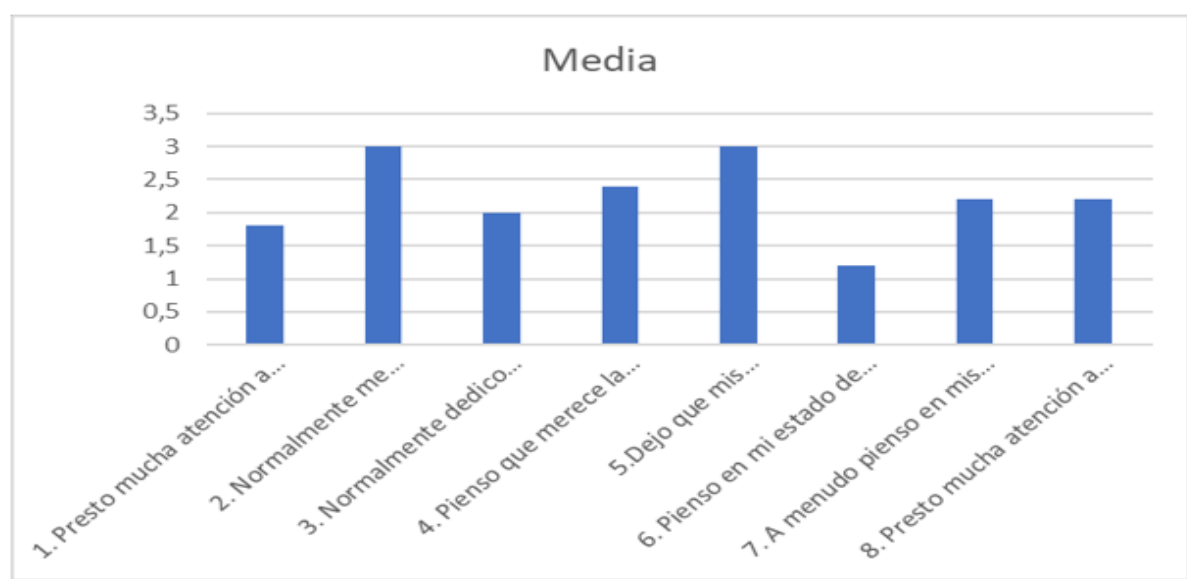

Fuente: Personas de la tercera edad del "Hogar de Acogimiento Del Adulto Mayor de San Pedro De Cevallos. Formularios en línea; IBM SPSS

La Dimensión de Atención Emocional, permite monitorear sí los adultos mayores prestan atención a sus propias emociones y sentimientos, así como a los de los demás; las frecuencias más relevantes son:

- "Normalmente me preocupo por lo que siento" con 15 adultos mayores respondieron Totalmente de acuerdo.

- "Dejo que mis sentimientos afecten a mis pensamientos" con 15 adultos mayores

- respondieron Muy de acuerdo. 
Dimensión de Claridad Emocional

\section{Tabla 6}

Escala de fiabilidad de la dimensión de claridad emocional

\begin{tabular}{c|cc}
\hline Alpha de Cronbach & Media & Items \\
\hline 0.92 & 78.39 & 8 \\
\hline
\end{tabular}

Fuente: Personas de la tercera edad del "Hogar de Acogimiento Del Adulto Mayor de San Pedro De Cevallos. Formularios en línea; IBM SPSS

Elaborado: Molina (2021)

La Dimensión de Claridad Emocional, es constituida por 8 elementos, evidenciándose un Alfa de Cronbach de 0,92, el cual conforme la tabla de escala de fiabilidad es "excelente", en cuanto a la media es de 78,38.

\section{Figura 2}

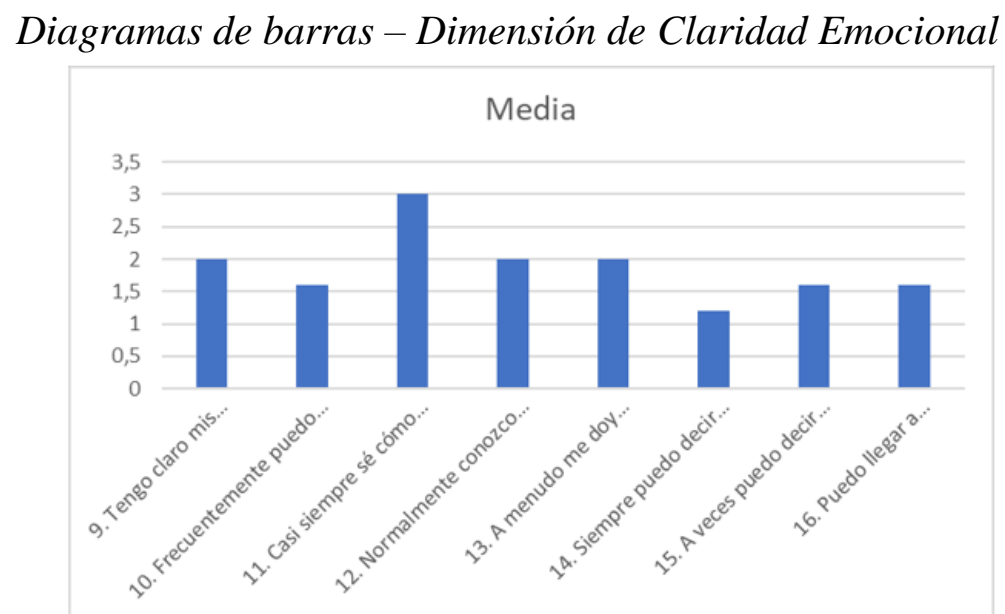

Fuente: Personas de la tercera edad del "Hogar de Acogimiento Del Adulto Mayor de San Pedro De Cevallos. Formularios en línea; IBM SPSS

Elaborado: Molina (2021)

La Dimensión de Claridad Emocional, permite identificar sí los adultos mayores perciben sus emociones propias y las de los demás; presentando énfasis en la pregunta "Casi siempre sé cómo me siento" con una frecuencia de Totalmente de acuerdo.

Dimensión de Reparación Emocional

Tabla 7

Estadísticas de Fiabilidad - Dimensión de Reparación Emocional

\begin{tabular}{c|c|c}
\hline Alpha de Cronbach & Media & Items \\
\hline 0.93 & 65.6 & 8 \\
\hline
\end{tabular}

Fuente: Personas de la tercera edad del "Hogar de Acogimiento Del Adulto Mayor de San Pedro De Cevallos. Formularios en línea; IBM SPSS

Elaborado: Molina (2021) 
La Dimensión de Claridad Emocional, es constituida por 8 elementos, evidenciándose un Alfa de Cronbach de 0,92, el cual conforme la tabla de escala de fiabilidad es "excelente", en cuanto a la media es de 65,6.

\section{Figura 3}

Diagrama de barras - Dimensión de Reparación Emocional

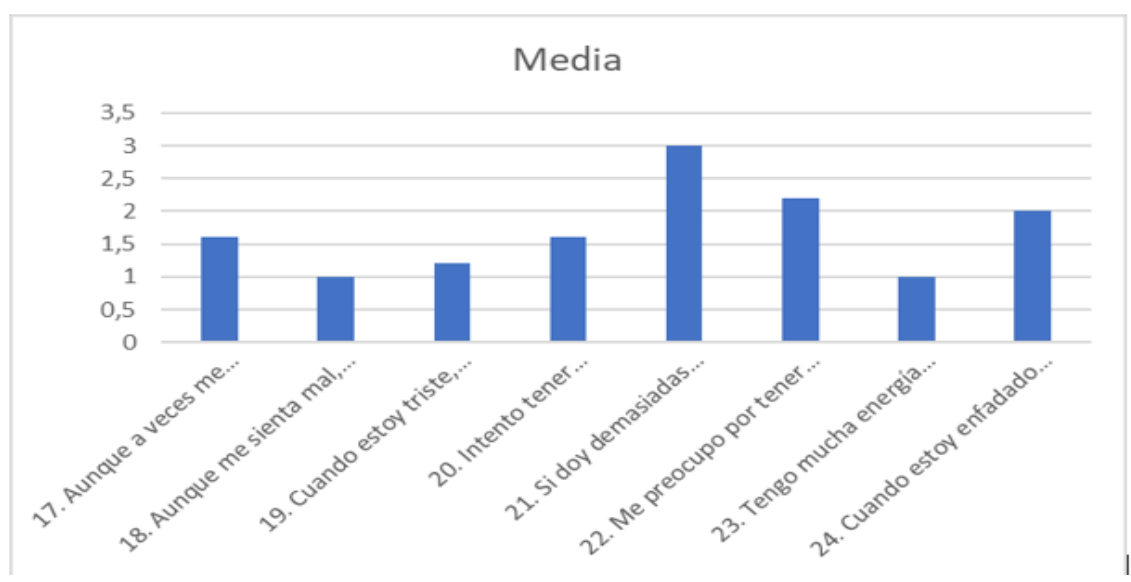

Fuente: Personas de la tercera edad del "Hogar de Acogimiento Del Adulto Mayor de San Pedro De Cevallos. Formularios en línea; IBM SPSS

Elaborado: Molina (2021)

La Dimensión de Reparación Emocional, permite observar sí los adultos mayores regulan los estados emocionales negativos y prolongan los positivos; tiene un grado de prevalencia en la pregunta “. Si doy demasiadas vueltas a las cosas, complicándolas, trato de calmarme" con una frecuencia de 15.

Análisis y discusión de los resultados de los resultados del CuestionarioWHOQOLBREF de calidad de vida

Tabla 8

Fiabilidad del Cuestionario WHOQOL-BREF de calidad de vida

\begin{tabular}{cccc}
\hline Dimensiones & Ítems & Correlación & $\begin{array}{c}\text { Alpha de } \\
\text { Cronoacn }\end{array}$ \\
\hline Física & 7 & 0,80 & 0,74 \\
Psicológica & 6 & 0,83 & 0,70 \\
$\begin{array}{c}\text { Relaciones } \\
\text { Sociales }\end{array}$ & 3 & 075 & 079 \\
Ambientales & 8 & 0,78 & 0,73 \\
Escala del & 24 & & 0,88 \\
Cuestionario & & &
\end{tabular}

Fuente: Molina (2021) 
El Cuestionario WHOQOL-BREF de calidad de vida contiene 24 elementos que fueron aplicados a 20 personas de la tercera edad del "Hogar de Acogimiento Del Adulto Mayor de San Pedro De Cevallos" evidenciándose un Alfa de Cronbach de 0,88 el cual conforme la tabla de escala de fiabilidad es excelente.

A continuación, se realiza un análisis de las 4 dimensiones que evalúa el test:

Dimensión Física

\section{Figura 4}

Diagrama de barras - Dimensión Física

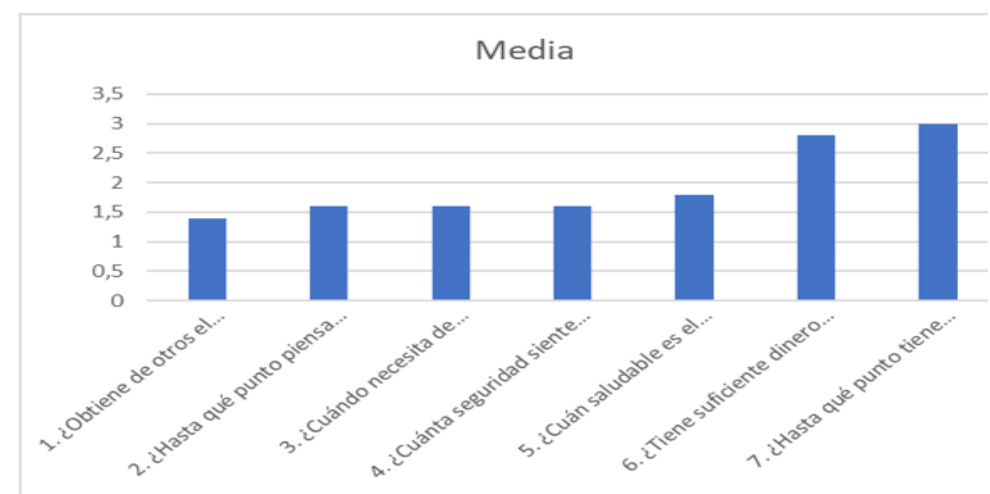

Fuente: Molina (2021)

La Dimensión Física, permite observar sí los adultos mayores tienen un adecuado estado físico; presentando relevancia en la pregunta:

- ¿Hasta qué punto tiene oportunidad para realizar actividades de ocio? con una frecuencia de 15.

\section{Dimensión Psicológica}

Figura 5

Diagrama de barras - Dimensión Psicológica

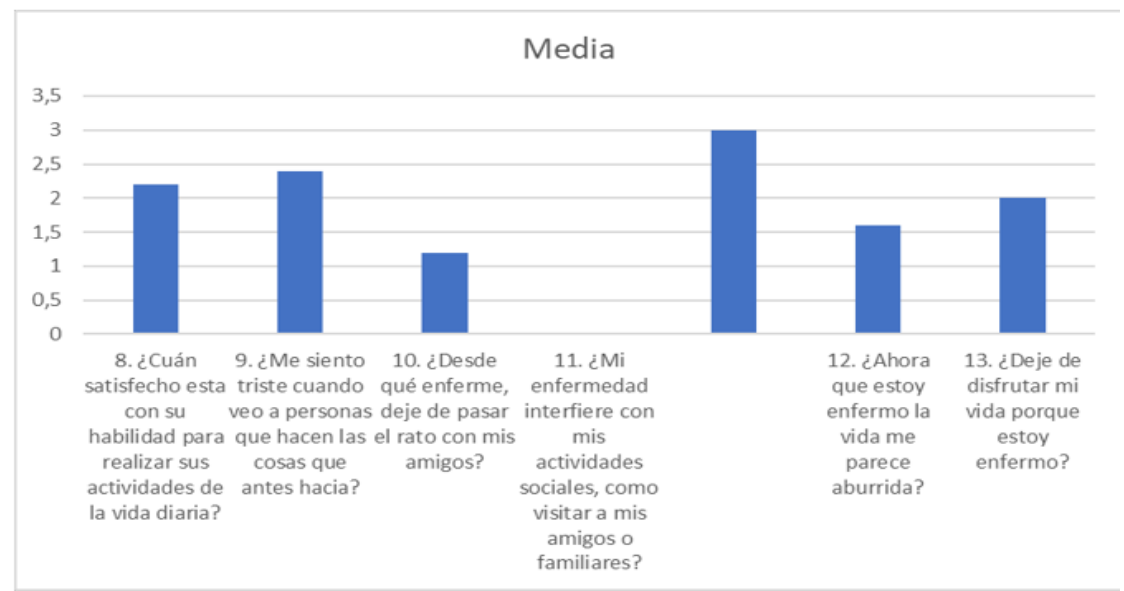

Fuente: Molina (2021) 
La Dimensión Psicológica, permite observar sí los adultos mayores tienen un adecuado estado psicológico; presentando relevancia en la pregunta: 11 ¿Mi enfermedad interfiere con mis actividades sociales, como visitar a mis amigos o familiares?

Dimensión Relaciones Sociales

\section{Figura 6}

Diagramas de barras - Relaciones Sociales

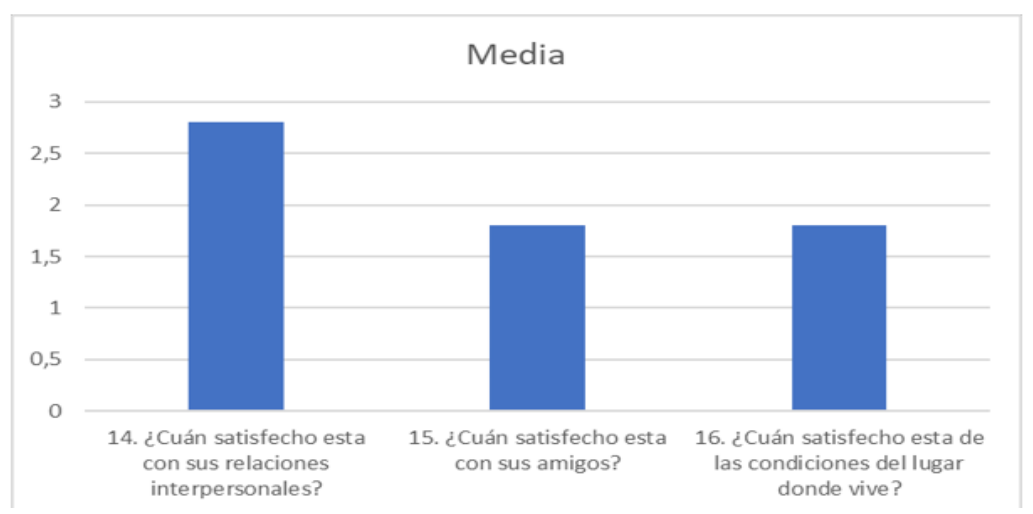

Fuente: Molina (2021)

La Dimensión de Relaciones Sociales, permite identificar sí los adultos mayores desarrollan un buen contacto interpersonal; presentando relevancia en la pregunta:

¿Cuán satisfecho esta con sus relaciones interpersonales? Con una frecuencia de Moderado 14.

\section{Dimensión Ambiental}

\section{Figura 7}

Diagramas de barras - Relaciones Sociales

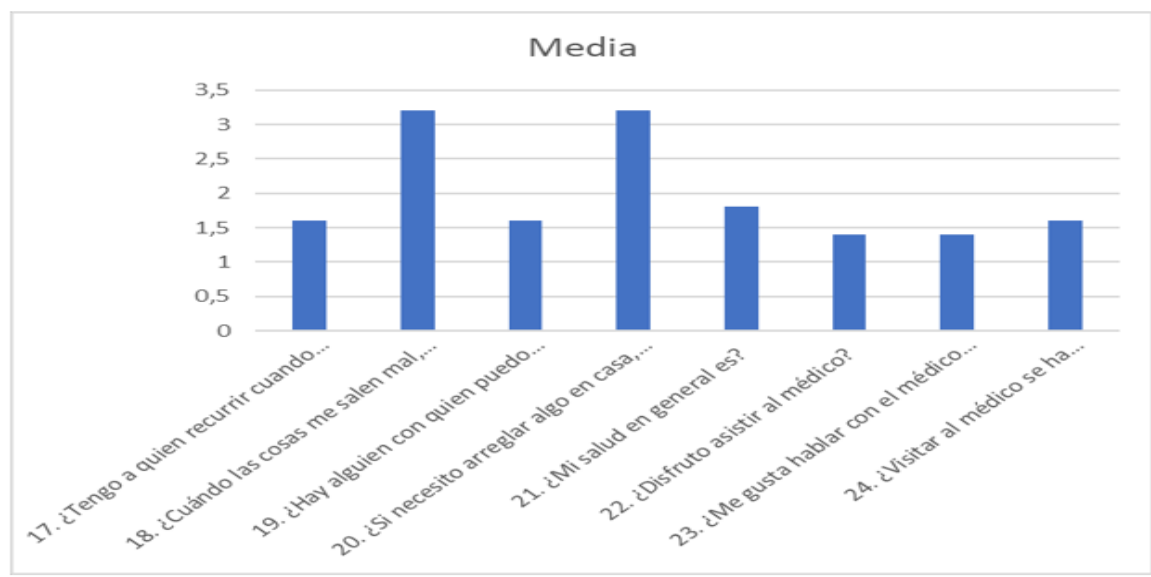

Fuente: Molina (2021) 
La Dimensión Ambiental, permite identificar sí las personas adultas mayores tienen un espacio correcto para desempeñar sus actividades, estas pueden ser de carácter lúdicas, físicas y de aseó; presentando relevancia en las siguientes preguntas:

- ¿Cuándo las cosas me salen mal, hay alguien que me puede ayudar? Con una Frecuencia de Bastante 16.

- ¿Si necesito arreglar algo en casa, hay alguien que me pueda ayudar? Con una frecuencia de Bastante 16.

\section{Verificación de hipótesis}

Para la verificación de la hipótesis se utilizó el Test TMMS-24 para medir la inteligencia emocional con una fiabilidad 0,93 Alpha de Cronbach y para medir la Variable Independiente se utilizó el Cuestionario WHOQOL-BREF de calidad de vida con una fiabilidad 0,88 Alpha de Cronbach, ayudándonos así a demostrar que la Inteligencia Emocional de los Adultos Mayores necesitan desarrollar su estabilidad en sus emociones para así ayudar a desarrollar una buena Calidad de Vida.

\section{Método de validación de hipótesis}

La investigación ha derivado en la obtención de datos cualitativos de escala ordinal para cadbariable. Para este tipo de datos pueden aplicarse estadísticos de asociación o decorrelación. En el caso de la asociación, la muestra de tamaño 20 no permite cumplircon la condición estadística para llenar la tabla de contingencia con máximo un $20 \%$ de celdas con frecuencias esperadas inferiores a 5. Por lo tanto, la aplicación del Chi cuadrado de Pearson no es viable. Esto quiere decir que, la opción adecuada para validar la hipótesis del presente estudio es la correlación mediante el coeficiente Tau- b de Kendall.

\section{Hipótesis estadísticas}

$\mathrm{H}_{0}$ : No hay correlación entre la inteligencia emocional y la calidad de vida de las personas de la tercera edad del "Hogar de Acogimiento el Adulto Mayor de San Pedro de Cevallos".

$\mathrm{H}_{1}$ : Hay correlación entre la inteligencia emocional y la calidad de vida de las personas de la tercera edad del "Hogar de Acogimiento el Adulto Mayor de San Pedro de Cevallos".

Nivel de significancia y regla de decisión

Con un nivel de significancia del 5\% se establece la siguiente regla de decisión:

$$
\text { H0: Pvalor (Sig. asintótica) > 0,05 }
$$


H1: Pvalor (Sig. asintótica) $\leq 0,05$

\section{Resultados}

Los resultados del cálculo llevado a cabo con el software SPSS 25 se muestran en la tabla 18.

\section{Tabla 9}

Tabla de correlaciones: Coeficiente Tau- $b$ de Kendall

\begin{tabular}{|c|c|c|c|c|c|}
\hline & & Física & Psicológica & $\begin{array}{c}\text { Relaciones } \\
\text { Sociales }\end{array}$ & Ambientales \\
\hline \multirow[t]{2}{*}{ Atención } & $\begin{array}{l}\text { Coeficiente } \\
\text { de } \\
\text { correlación }\end{array}$ & 0,054 & $-0,097$ & $-0,321$ & $-0,162$ \\
\hline & Sig. & 0,803 & 0,649 & 0,126 & 0,455 \\
\hline \multirow[t]{2}{*}{ Claridad } & $\begin{array}{l}\text { Coeficiente } \\
\text { de } \\
\text { correlación }\end{array}$ & $-0,118$ & $-0,381$ & 0,085 & 0,059 \\
\hline & $\begin{array}{c}\text { Sig. } \\
\text { (bilateral) }\end{array}$ & 0,579 & 0,069 & 0,680 & 0,781 \\
\hline \multicolumn{6}{|c|}{$\begin{array}{c}\text { Coeficiente } \\
\text { de } \\
\text { Reparación correlación }\end{array}$} \\
\hline & Sig. & 0,588 & 0,046 & 0,636 & 0,453 \\
\hline
\end{tabular}

Fuente: Molina (2021)

Los resultados del análisis de correlación muestran que, el valor de prueba (Sig. bilateral) es inferior al nivel de significancia $(0,05)$ únicamente entre las dimensiones reparación y psicología, con un coeficiente igual a 0,433. Esto indica que, la reparación y la psicología se correlacionan de forma directa, con una fuerza media.

\section{Discusión}

En el análisis de resultados se demuestra la correlación existente entre la reparación (dimensión relacionada a la calidad de vida) y la psicología (dimensión vinculada a la inteligencia emocional); es decir, se rechaza la hipótesis nula, y se acepta la hipótesis alterna que dice: Hay correlación entre la inteligencia emocional y la calidad de vida de las personas de la tercera edad del "Hogar de Acogimiento el Adulto Mayor de San Pedro de Cevallos". 


\section{Conclusiones}

- En el presente estudio se determina que la Inteligencia Emocional influyo en la Calidad de vida Durante la pandemia Covid-19 en las personas de la tercera edad en el "Hogar de Acogimiento del Adulto Mayor de San Pedro de Cevallos", en los componentes de Atención, Claridad y Reparación.

- Se pudo establecer de manera bibliografía y documental que las emociones se desarrollan en el Adulto Mayor favoreciendo el desarrollo cognoscitivo en función de la solución de nuevos problemas.

- Tras la aplicación del Test propuesto se observó que el estado emocional de los Adultos Mayores es bastante estable no obstante se identificó dificultades que se reflejan en las siguientes; dimensiones de Atención Emocional y Claridad Emocional. Así también, y en la Aplicación del Cuestionario WHOQOL-BREF se determinó que se encuentra con una calidad de vida estable; sin embargo, es necesario trabajar en la Dimensión Psicológica específicamente en la depresión que se observa en los Adultos Mayores.

- Para finalizar se desarrolló el Diseño de una guía metodológica sobre La Resiliencia para las personas de la tercera edad del Hogar de Acogimiento del Adulto Mayor del Cantón Cevallos, la misma que se realizó a través de investigaciones bibliografías, y basadas en los resultados presentes en las técnicas de observación utilizadas.

\section{Referencias bibliográficas}

Estrada Guerrero, R., Lemus Torres, D., Mendoza Anaya, D., \& Rodríguez Lugo, V. (2010). Hidrogeles poliméricos potencialmente aplicables en Agricultura.

Lucas, R. (1998). Versión Española del WHOQOL. Madrid: Ergón.

Molina, D. (2021). La inteligencia emoción y su calidad de vida durante la pandemia Covid.19 en personas de la tercera edad del hogar de acogimiento del adulto mayor del cantón Cevallos.

Velongia. (1994). Investigación en salud y calidad de vida. Bogotá.

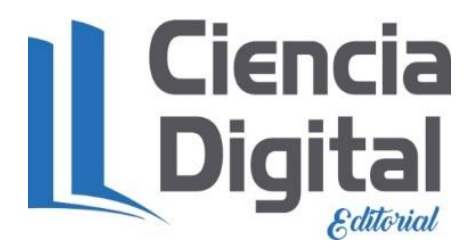


El artículo que se publica es de exclusiva responsabilidad de los autores y no necesariamente reflejan el pensamiento de la Revista Conciencia Digital.

\section{Ciencia
Digital
Ethtoul}

El artículo queda en propiedad de la revista y, por tanto, su publicación parcial y/o total en otro medio tiene que ser autorizado por el director de la Revista Conciencia Digital.
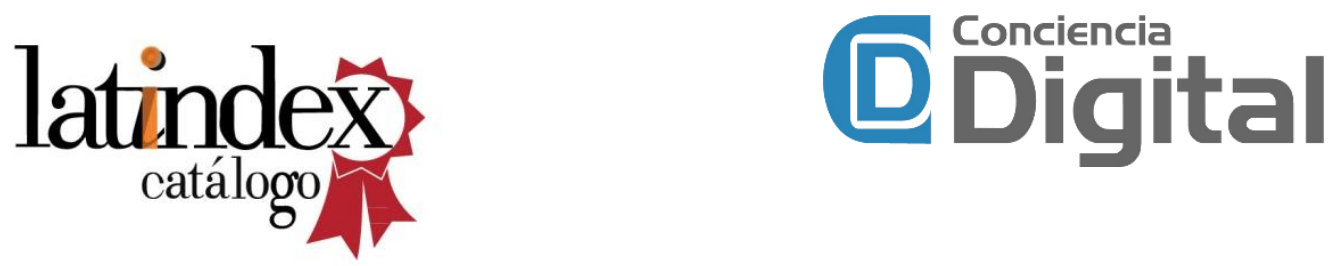

Indexaciones

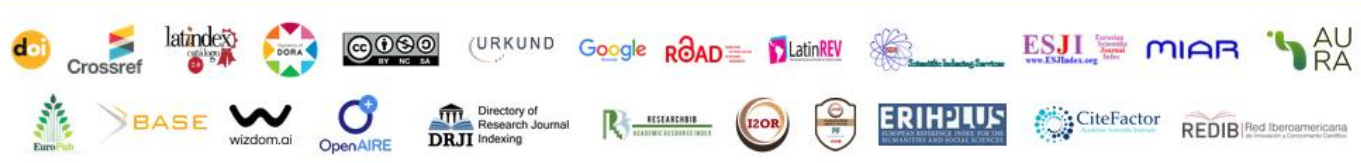

